\title{
Precise Segmentation of Multiple Organs in CT Volumes Using Learning-Based Approach and Information Theory
}

\author{
Chao Lu $^{1,3}$, Yefeng Zheng ${ }^{1}$, Neil Birkbeck ${ }^{1}$, Jingdan Zhang ${ }^{1}$, Timo Kohlberger ${ }^{1}$, \\ Christian Tietjen ${ }^{2}$, Thomas Boettger ${ }^{2}$, James S. Duncan ${ }^{3}$, and S. Kevin Zhou ${ }^{1}$ \\ 1 Image Analytics and Informatics, Siemens Corporate Research, Princeton, New Jersey, USA \\ 2 Computed Tomography, Siemens Healthcare, Forchheim, Germany \\ 3 School of Engineering \& Applied Science, Yale University, New Haven, Connecticut, USA
}

\begin{abstract}
In this paper, we present a novel method by incorporating information theory into the learning-based approach for automatic and accurate pelvic organ segmentation (including the prostate, bladder and rectum). We target $3 \mathrm{D}$ CT volumes that are generated using different scanning protocols (e.g., contrast and non-contrast, with and without implant in the prostate, various resolution and position), and the volumes come from largely diverse sources (e.g., diseased in different organs). Three key ingredients are combined to solve this challenging segmentation problem. First, marginal space learning (MSL) is applied to efficiently and effectively localize the multiple organs in the largely diverse CT volumes. Second, learning techniques, steerable features, are applied for robust boundary detection. This enables handling of highly heterogeneous texture pattern. Third, a novel information theoretic scheme is incorporated into the boundary inference process. The incorporation of the Jensen-Shannon divergence further drives the mesh to the best fit of the image, thus improves the segmentation performance. The proposed approach is tested on a challenging dataset containing 188 volumes from diverse sources. Our approach not only produces excellent segmentation accuracy, but also runs about eighty times faster than previous state-of-the-art solutions. The proposed method can be applied to CT images to provide visual guidance to physicians during the computer-aided diagnosis, treatment planning and image-guided radiotherapy to treat cancers in pelvic region.
\end{abstract}

\section{Introduction}

Automated segmentation of medical images has increasingly become a valuable tool for medical image analysis. Pelvic region analysis plays an important role in the medical diagnosis and treatment planning for prostate cancer and bladder cancer. Segmentation of prostate as well as bladder and rectum from a three dimensional computed tomography (CT) volume often serves as the first step in image-based radiotherapy studies and

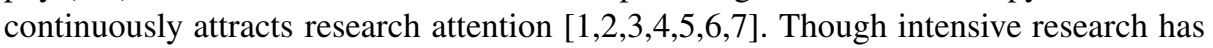
been performed, accurate segmentation of 3D soft tissue structures in pelvic region is still a challenging problem, due to the large variations in organ shapes and in the texture pattern inside and along organ boundaries. 


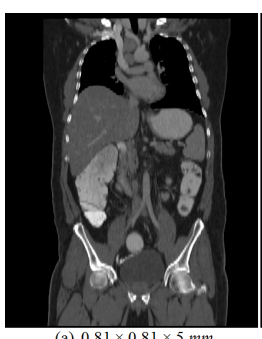

(a) $0.81 \times 0.81 \times 5 \mathrm{~mm}$

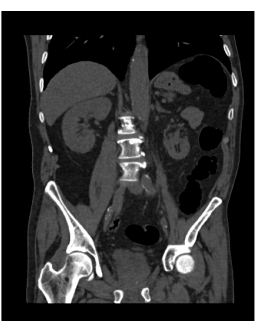

(b) $1.0 \times 1.0 \times 1.0 \mathrm{~mm}$

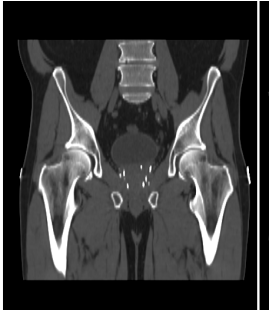

(c) $0.98 \times 0.98 \times 3 \mathrm{~mm}$

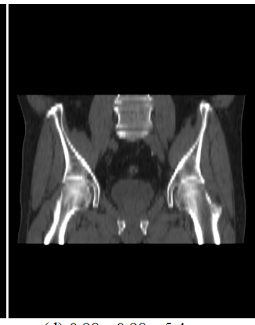

(d) $0.98 \times 0.98 \times 5.4 \mathrm{~mm}$

Fig. 1. Examples of CT volumes in our dataset. Note the variance in the image size, position of prostate / bladder / rectum, and volume dimensions. (c) also presents a challenging case that the patient is undergoing brachytherapy, with implanted metal seeds in the prostate.

Unlike in previous work, the CT volumes in our study are scanned under largely diverse protocols. Specifically, there are several diversities in our dataset: 1) The volume dimension and organ position in the volume substantially vary; 2) Some volumes are enhanced by contrast agent while some are not; 3) Some volumes come from the patients undergoing brachytherapy with implanted metal seeds in the prostate; 4) The inter-slice resolution changes from $0.8 \mathrm{~mm}$ to $5.0 \mathrm{~mm}$. Some examples are shown in Figure 1. These diversities increase the variability of the key organs in both shape and texture patterns, and therefore render the problem more challenging. Furthermore, the automatic detection (or localization) is made more difficult especially because of 1). Albeit important, automatic object detection is largely ignored in previous work.

In this paper, we propose an automatic segmentation approach that addresses all the challenges mentioned above. Figure 2 illustrates the flowchart of the proposed method. There are three major contributions in our system. First, the system uses a marginal space learning strategy, which efficiently and effectively solves the organ detection problem. Second, a learning-based boundary localization technique is utilized. By using this technique, the system not only achieves accurate boundary responses, but also becomes reliable to the heterogeneous texture patterns. Third, an information theory based module is incorporated into the framework and the Jensen-Shannon divergence-based uncertainty measures are used for further improvement.

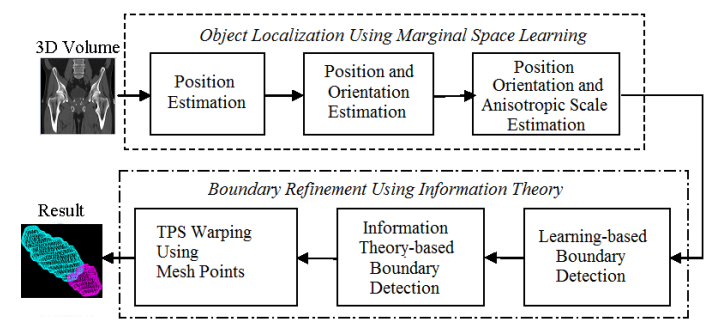

Fig. 2. Diagram for the proposed method 


\section{Organ Detection and Mesh Initialization - Hierarchical Detection Network}

We represent the shape of an organ by a dense collection of mesh points on its boundary, therefore, by a closed triangle mesh. In our work, the prostate is constructed with 602 points, while the bladder and rectum are represented using 1202 and 962 points, respectively.

We initialize the multi-organ segmentation from an existing learning-based detection framework. In the first stage, we performed a hierarchical bounding box detection system based on Marginal Space Learning [8] in connection with Probabilistic Boosting Trees [9]. The latter yields bounding box estimates for the prostate, the bladder, and the rectum.

After automatic object localization, we align the mean shape with the estimated pose onto the 3D volume, which is used as the initialization for mesh detection. An illustration of mesh initialization for bladder is presented in Figure 3 .
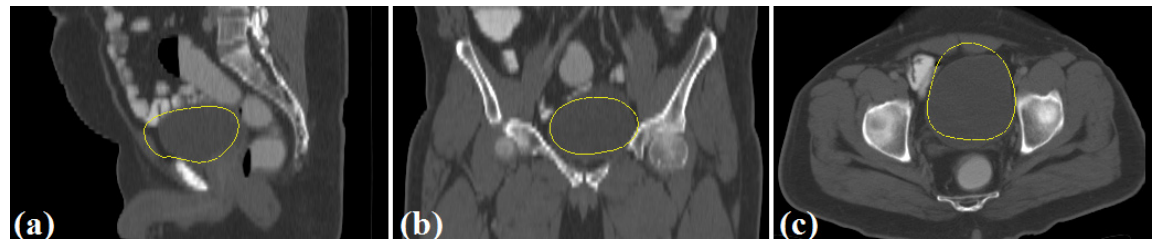

Fig. 3. Mesh initialization after pose detection for the bladder. (a)(b)(c) show the sagittal, coronal and axial view, respectively.

\section{Boundary Refinement}

After initialization, we then perform the boundary refinement to accurately fit the object boundary. Active shape models (ASM) are widely used to deform an initial estimate of a deformable shape under the guidance of the image evidence and the shape prior. However, the generic boundary detector in the original ASM does not work in our application due to the complicated background and weak edges. There are two key components in our refinement method: probabilistic boundary response, and information theoretic measure (Jensen-Shannon divergence).

\subsection{Learning-Based Boundary Detection}

A key issue in boundary refinement is boundary localization, which usually involves locally searching around current shape boundaries. Specifically, for a current boundary point $p \in P$, a candidate point set $Q_{p}$ is formed by including points along the normal direction at $p$ and within some distance. Then the point in $Q_{p}$ with the maximum boundary probability (response) is used to replace $p$, i.e.,

$$
p \leftarrow \arg \max _{q \in Q_{p}} \operatorname{Pr}(\text { boundary } \mid q, \text { vol }) .
$$


Previous work usually approximates the boundary response by simple checking gradients or intensity distribution along surface normals. However, the information gathered this way is not enough for our task because the texture pattern in the pelvic region has a large variability. When dealing with data from different scanning protocols, the variability is even larger.

In order to attack this problem, we decide to learn $\operatorname{Pr}($ boundary $\mid q, \mathrm{vol})$ from the training set. In this paper, we train a boundary detector using our efficient steerable features and PBT, similarly to [8]. The same approach is used to train a boundary detector using the PBT [9] and steerable features. According to our experiments, the learning-based approach provides a robust boundary inference and it is reliable to the heterogeneous texture patterns, however, there is still large potential for improving the precision.

\subsection{Information Theory Incorporation}

In soft-tissue segmentation, there is often no clear boundary information. The intensity gradient at the interface of adjacent tissues is also low. To overcome the difficulty, besides the learning-based method, we also incorporate the information theory based class-uncertainty information into our boundary refinement work, using JensenShannon divergence measure.

Jensen-Shannon (JS) divergence, first introduced in [10], serves as a measure of cohesion between multiple probability distributions. It has been used as a dissimilarity measure for image registration with promising results. It has several desirable properties, to name a few, 1) The square root of JS-divergence is a metric [11]; 2) JSdivergence relates to other information theoretic functionals, such as the relative entropy or the Kullback divergence, and hence it shares their mathematical properties as well as their intuitive appeal; 3) The compared distributions using the JS-divergence can be weighted, which allows one to take into account the different sizes of the point set samples from which the probability distributions are computed; 4) the JS-divergence is bounded by 0 and 1 .

In this paper, the JS-divergence is the main measure that we incorporate into the boundary refinement framework. Given a priori knowledge of intensity probability distributions of each object, the JS-divergence can be computed for each intensity value, which yields the uncertainty level of the classification. Suppose the image is divided into $m$ objects, denoted by $o_{i}, i \in[0,1, \ldots, m-1]$. For any candidate point $q$ in $Q_{p}$ with image intensity $I(q)$, the posteriori probability $p\left(o_{i} \mid I\right)$ is obtained from the training set using Bayes rules:

$$
P_{i}(I)=p\left(o_{i} \mid I\right)=\frac{p\left(I \mid o_{i}\right) \cdot p\left(o_{i}\right)}{p(I)},
$$

where $p\left(I \mid o_{i}\right), p\left(o_{i}\right)$, and $p(I)$ are learned from the training set.

The JS-divergence of each intensity $I$ is defined by:

$$
\begin{aligned}
J S(I) & =J S\left(P_{1}(I), P_{2}(I), \ldots, P_{i}(I)\right)=H\left(\Sigma \pi_{i} P_{i}(I)\right)-\Sigma \pi_{i} H\left(P_{i}(I)\right) \\
& =H\left(\Sigma \pi_{i} p\left(o_{i} \mid I\right)\right)+\Sigma\left(\pi_{i} \cdot p\left(o_{i} \mid I\right) \log \left(p\left(o_{i} \mid I\right)\right)\right),
\end{aligned}
$$


where $\pi=\left\{\pi_{1}, \pi_{2}, \ldots, \pi_{n} \mid \pi_{i}>0, \Sigma \pi_{i}=1\right\}$ are the weights of the probability distributions $P_{i}$, and $H(\mathbf{x})$ is the Shannon entropy, i.e., $H(x)=\int-p(x) \log p(x) d x$. The two terms on the right hand side are the entropy of $\mathbf{P}=\Sigma \pi_{i} P_{i}(I)$ (the $\pi$-convex combination of the $P_{i} \mathrm{~s}$ ) and the same convex combination of the respective entropies.

In [12], the authors defined a simple uncertainty measure, which is a specific case of our general Jensen-Shannon divergence, but the mathematical properties demonstrated in [12] still hold for JS divergence. Intensities with high JS-divergence measures tend to appear close to object boundaries rather than inside homogeneous regions. Combined with the gradient information, the JS-divergence measure is expected to provide an enhanced on-surface cost at the locations absent a clear boundary. Let $\nabla(q)$ denote the gradient magnitude at a candidate point $q$, our information theoretic measure has the following form:

$$
\begin{aligned}
p & \leftarrow \arg \max _{q \in Q_{p}} C(I(q), \text { vol }) \\
& =\arg \max _{q \in Q_{p}}[\nabla(q)+\alpha J S(q)],
\end{aligned}
$$

where $\alpha$ is a constant parameter. Here we set $\alpha=0.5$ according to the experiments on the training set. For simplicity, we choose $\pi_{i}=p\left(o_{i}\right)$, and in this paper, the volume is divided into four parts: prostate, bladder, rectum and the background. It is straightforward to see that $\Sigma \pi_{i}=1$.

After performing the control point adjustment described above, the mesh points fit the boundary well, but the contour may not be smooth. Then, we project the deformed shape onto a shape subspace. In all our experiments, to determine the dimension of the subspace, we demand it to capture $95 \%$ variations. As shown in previous work [8], the statistical shape model is very effective to enforce the prior shape constraint.

\section{Experiments}

\subsection{Dataset}

Our database contains 188 3D CT volumes, each with three annotated groundtruth dense meshes for prostate, bladder and rectum, respectively. As mentioned in the introduction, the dataset is very challenging in that the volumes come from largely diverse sources. This heterogeneity causes a large variation in both shape deformation and texture patterns of the organs. Moreover, diagnosis of different diseases often request different contrast agent to be injected into the patient, or no contrast at all.

\subsection{Evaluation}

We conducted the evaluation using a four-fold cross validation. Errors are measured using the average symmetric surface distance. The average running time for one volume, including all steps, is around 1.06 seconds for the prostate, 1.51 seconds for the bladder and 2.53 seconds for the rectum (on an Intel 8-Core $2.00 \mathrm{GHz}$ processor, with 16G RAM). 
Figure 4 illustrates a difficult case that the patient is undergoing brachytherapy in prostate. The learning-based approach cannot lock on the edge of prostate while the proposed method produces satisfying result. The comparison of bladder segmentation is also shown in Figure 4.

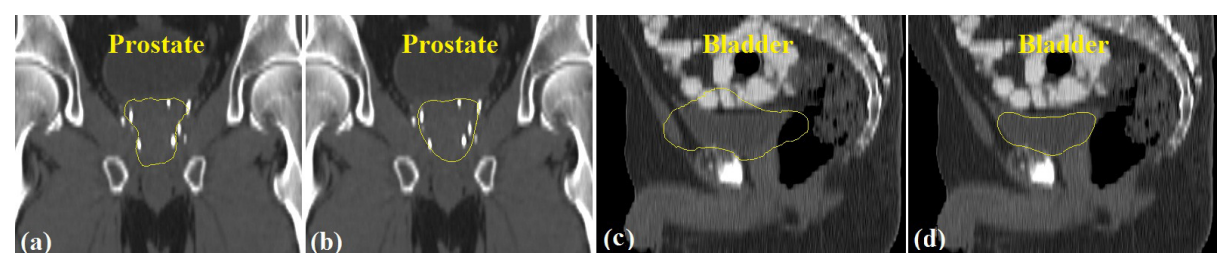

Fig. 4. Segmentation of the prostate and bladder, using (a)(c) the learning-based approach only, and (b)(d) the proposed method

To give a thorough evaluation, in this paper, we use four-fold cross validation to evaluate our algorithm. Specifically, the whole dataset (188 cases) is divided to four sets, each containing 47 volumes. Each time one set is chosen as the testing set and the rest as the training set (for both the shape model and boundary classifiers). This is done for four times and the average performance is reported.

The evaluation results for cross validation are summarized in Table 1 The mean error measured in the average symmetric surface distance is $2.37 \mathrm{~mm}$ for the prostate, and the median is $2.15 \mathrm{~mm}$. For the bladder, the mean and median error are $2.81 \mathrm{~mm}$ and $2.24 \mathrm{~mm}$, respectively. The rectum achieves an accuracy of $4.23 \mathrm{~mm}$ and $4.09 \mathrm{~mm}$ for the mean and median, respectively.

Table 1. Cross Validation for the Proposed Method (mm)

\begin{tabular}{|c|c|c|c|c|c|c|c|}
\hline & Method & Mean & STD & Median & Minimum & Maximum & $80^{t h}$ percentile \\
\hline Prostate & Learning-based [8] & 3.53 & 2.23 & 3.00 & 1.52 & 13.06 & 5.01 \\
& Proposed Method & 2.37 & 0.89 & 2.15 & 1.10 & 5.58 & 2.96 \\
\hline \multirow{3}{*}{ Bladder } & Learning-based [8] & 3.89 & 1.89 & 3.70 & 0.87 & 10.39 & 5.53 \\
& Proposed Method & 2.81 & 1.86 & 2.24 & 0.82 & 8.76 & 3.38 \\
\hline \multirow{2}{*}{ Rectum } & Learning-based [8] & 6.44 & 1.71 & 6.19 & 3.65 & 12.46 & 7.86 \\
& Proposed Method & 4.23 & 1.46 & 4.09 & 1.81 & 8.19 & 5.24 \\
\hline
\end{tabular}

It is difficult to directly compare different segmentation approaches due to the use of different datasets as well as different annotations. However, it is worth summarizing the previous experiments to comprehend the status of the study. Table 2 provides a brief summary of recent works on automatic pelvic region segmentation with reported average surface distances along with datasets used. The table shows clearly that our precision is among the the best reported. Compared to the method [1] with similar reported precisions, our approach have two apparent strengths. First, the dataset used in our experiments has more diversities than previous tested datasets. Second, our method is more efficient, i.e. runs more than eighty times faster. 
Table 2. Comparison with Recent Work on Prostate Segmentation

\begin{tabular}{|c|c|c|c|c|c|c|c|}
\hline Method & Mean Error & Speed & Data set & Auto? & Interslice Res. & Implanted? & Organs \\
\hline Freedman et al. [5] & $3.91 \mathrm{~mm}$ & NA & 48 & Yes & NA & No & P, R \\
\hline Rousson et al. [3] & 4 to $5 \mathrm{~mm}$ & $12 \mathrm{~s}$ & 16 & Semi & $3 \mathrm{~mm}$ & No & P, B \\
\hline Feng et al. [1] & $2.47 \mathrm{~mm}$ & $96 \mathrm{~s}$ & 24 & Semi & $3 \mathrm{~mm}$ & No & P \\
\hline Proposed Method & $2.37 \mathrm{~mm}$ & $1.06 \mathrm{~s}$ & 188 & Yes & Varies & Mixed & P, B, R \\
\hline
\end{tabular}

Some typical segmentation results are shown in Figure 5 from three orthogonal views, with segmented prostate in yellow, bladder in blue and rectum in green.

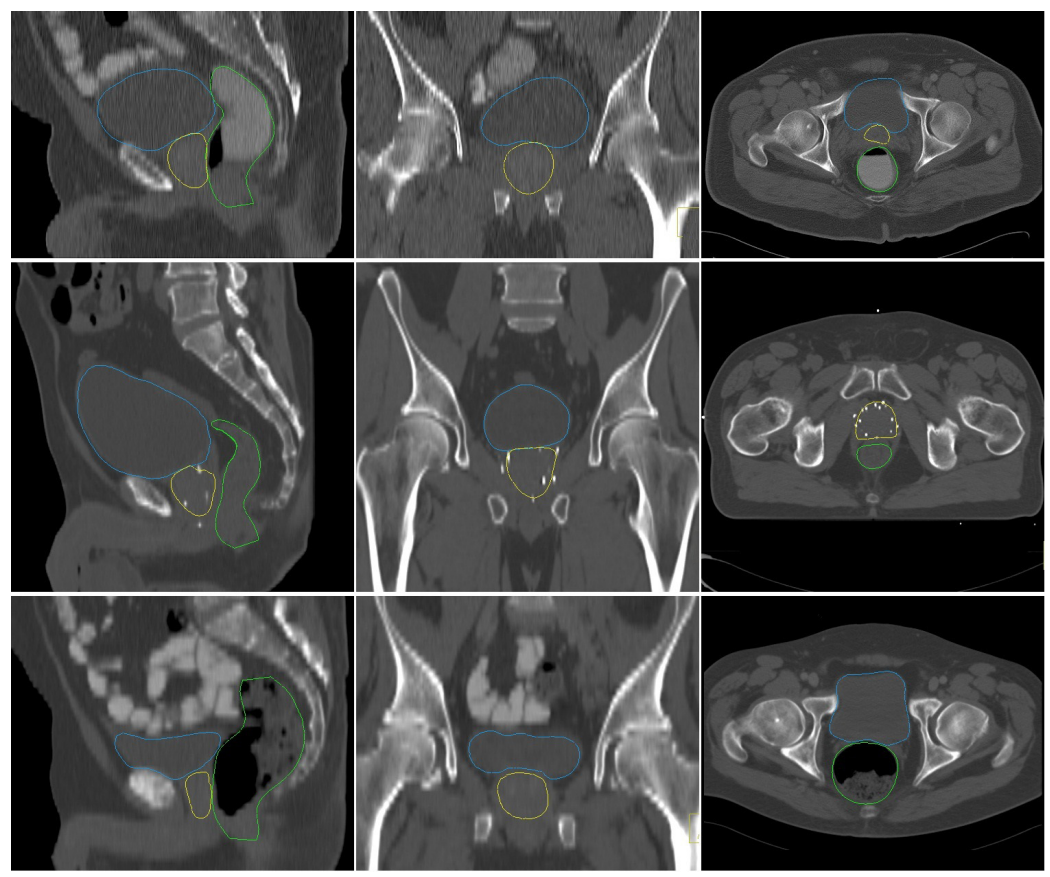

Fig. 5. Examples of pelvic-region multiple organ segmentation, with yellow for the prostate, blue for the bladder, and green for the rectum. From left to right: sagittal, coronal, and axial slices.

\section{Conclusion}

In this paper, we proposed a novel approach that incorporates information theory with the learning-based approach, for automatic pelvic-region multiple organ segmentation from a 3D CT volume. We target on general data from patients with different diseased organs and scanned under different protocols. Despite the challenges, our approach demonstrates robust performance in accuracy and runs more efficiently than state-ofthe-art solutions. Using the techniques described above, it is possible for the physicians to efficiently delineate the key pelvic organs for diagnosis and treatment planning, and also precisely guide the interventional devices toward the organs during image-guided radiotherapy. 


\section{References}

1. Feng, Q., Foskey, M., Chen, W., Shen, D.: Segmenting CT prostate images using population and patient-specific statistics for radiotherapy. Medical Physics 37(8), 4121-4132 (2010) $462,467,468$

2. Lu, C., Chelikani, S., Papademetris, X., Knisely, J.P., Milosevic, M.F., Chen, Z., Jaffray, D.A., Staib, L.H., Duncan, J.S.: An integrated approach to segmentation and nonrigid registration for application in image-guided pelvic radiotherapy. Medical Image Analysis 15(5), 772-785 (2011) 462

3. Rousson, M., Khamene, A., Diallo, M., Celi, J.C., Sauer, F.: Constrained Surface Evolutions for Prostate and Bladder Segmentation in CT Images. In: Liu, Y., Jiang, T., Zhang, C. (eds.) CVBIA 2005. LNCS, vol. 3765, pp. 251-260. Springer, Heidelberg (2005) 462468

4. Lu, C., Duncan, J.S.: A coupled segmentation and registration framework for medical image analysis using robust point matching and active shape model. In: 2012 IEEE Workshop on Mathematical Methods in Biomedical Image Analysis (MMBIA), pp. 129-136 (January 2012) 462

5. Freedman, D., Radke, R., Zhang, T., Jeong, Y., Lovelock, D., Chen, G.: Model-based segmentation of medical imagery by matching distributions. IEEE Transactions on Medical Imaging 24(3), 281-292 (2005) 462, 468

6. Lu, C., Chelikani, S., Jaffray, D.A., Milosevic, M.F., Staib, L.H., Duncan, J.S.: Simultaneous nonrigid registration, segmentation, and tumor detection in MRI guided cervical cancer radiation therapy. IEEE Transactions on Medical Imaging 31(6), 1213-1227 (2012) 462

7. Seifert, S., Barbu, A., Zhou, S.K., Suehling, M., Cavallaro, A., Comaniciu, D.: Hierarchical parsing and semantic navigation of full body ct data. In: SPIE Medical Imaging (February 2009) 462

8. Zheng, Y., Barbu, A., Georgescu, B., Scheuering, M., Comaniciu, D.: Four-chamber heart modeling and automatic segmentation for 3-D cardiac CT volumes using marginal space learning and steerable features. IEEE Trans. on Med. Imaging 27(11), 1668-1681 (2008) $464,465,466,467$

9. Tu, Z.: Probabilistic boosting-tree: learning discriminative models for classification, recognition, and clustering. In: ICCV 2005, vol. 2, pp. 1589-1596 (October 2005) 464465

10. Lin, J.: Divergence measures based on the Shannon entropy. IEEE Trans. Infor. Theory 37, 145-151 (1991) 465

11. Endres, D., Schindelin, J.: A new metric for probability distributions. IEEE Trans. Inf. Theory 49, 1858-1860 (2003) 465

12. Saha, P., Udupa, J.: Optimum threshold selection using class uncertainty and region homogeneity. IEEE Trans. Pattern Anal. Machine Intell. 12, 689-706 (2001) 466 